\title{
Survival of halophilic Archaea in Earth's cold stratosphere
}

\author{
P. DasSarma', V.J. Laye', J. Harvey², C. Reid ${ }^{2}$, J. Shultz², A. Yarborough ${ }^{2}$, A. Lamb ${ }^{2}$, \\ A. Koske-Phillips' ${ }^{2}$ A. Herbst ${ }^{2}$, F. Molina ${ }^{2}$, O. Grah'², T. Phillips ${ }^{2}$ and S. DasSarma' \\ ${ }^{1}$ Department of Microbiology and Immunology, Institute of Marine and Environmental Technology, University of Maryland \\ School of Medicine, Baltimore, MD 21202,USA e-mail: pdassarma@som.umaryland.edu \\ ${ }^{2}$ Earth to Sky Calculus and Spaceweather.com, Bishop, CA, USA
}

\begin{abstract}
Halophilic Archaea are known to tolerate multiple extreme conditions on Earth and have been proposed as models for astrobiology. In order to assess the importance of cold-adaptation of these microorganisms in surviving stratospheric conditions, we launched live, liquid cultures of two species, the mesophilic model Halobacterium sp. NRC-1 and the cold-adapted Antarctic isolate Halorubrum lacusprofundi ATCC 49239, on helium balloons. After return to Earth, the cold-adapted species showed nearly complete survival while the mesophilic species exhibited slightly reduced viability. Parallel studies found that the cold-adapted species was also better able to survive freezing and thawing in the laboratory. Genome-wide transcriptomic analysis was used to compare the two haloarchaea at optimum growth temperatures versus low temperatures supporting growth. The cold-adapted species displayed perturbation of a majority of genes upon cold temperature exposure, divided evenly between up-regulated and downregulated genes, while the mesophile exhibited perturbation of only a fifth of its genes, with nearly two-thirds being down-regulated. These results underscore the importance of genetic responses of $H$. lacusprofundi to cold temperature for enhanced survival in the stratosphere.
\end{abstract}

Received 22 August 2016, accepted 15 September 2016, first published online 27 October 2016

Key words: cold temperature, extremophiles, Halobacterium sp. NRC-I, Halorubrum lacusprofundi, helium balloon, Mars analogue, stratosphere.

\section{Introduction}

The recent discovery of brine flows on the surface of Mars has intensified interest in the extremophilic character of extremely halophilic microorganisms in relation to astrobiology (Mancinelli et al. 2004; DasSarma 2006; Horneck et al. 2010; Leuko et al. 2014). Flowing brines were first noted as seasonal dark streaks or recurring slope lineae (RSL) on the walls of Garni crater observed from images captured by NASA's Mars Reconnaissance Orbiter (McEwen et al. 2011). The occurrence of RSLs at subzero temperatures indicated that frozen brines melt and flow seasonally on the Martian surface (Ojha et al. 2015). This prediction was also supported by spectroscopic evidence for hydrated sodium and magnesium chloride, chlorate and perchlorate salts at the Phoenix lander site (Kounaves et al. 2014; Toner et al. 2014). Interest in Mars Special Regions, such as the Phoenix site, is heightened by the existence of usable metabolic energy and its potential for enabling habitability (Stoker et al. 2010; Schuerger et al. 2013; Rummel et al. 2014).

On Earth, hypersaline brines are nearly ubiquitous and generally thalassic, with high biological productivity promoted by sunlight driving pigment production and a variety of primary metabolic processes. A diversity of halophilic microorganisms flourish, originating from all three branches of life: Archaea, Bacteria and Eukarya (DasSarma \& DasSarma 2012). Halophilic Archaea (Haloarchaea) are able to tolerate high salinity primarily through the salt-in strategy, producing negatively charged proteins that remain soluble and compete successfully with ions for hydration (Karan et al. 2012; DasSarma \& DasSarma 2015, see also Goh et al. 2011). Bacteria usually survive by the salt-out strategy, synthesizing or taking-up zwitterionic compatible solutes, which helps maintain osmotic balance with external salts, while Eukarya generally produce neutral polyols, e.g. glycerol, for the same purpose (DasSarma \& DasSarma 2012). Combinations of these mechanisms operate in some species (DasSarma \& DasSarma 2015).

Among the Haloarchaea, Halobacterium sp. NRC-1 has been extensively studied for its extremophilic character (DasSarma et al. 2006; DasSarma \& DasSarma 2012). This species is capable of tolerating high concentrations of sodium and potassium chlorides and perchlorates (Coker et al. 2007; DasSarma et al. unpublished). In addition to high salinity, this strain is slightly thermotolerant with optimum growth at $42^{\circ} \mathrm{C}$ and survival at $49-50^{\circ} \mathrm{C}$ (Coker et al. 2007) and is highly resistant to ultraviolet (UV) and ionizing radiation (McCready et al. 2005; DeVeaux et al. 2007). Genetic, genomic and transcriptomic studies have established a wide range of survival mechanisms operating in Halobacterium, including presence of highly acidic proteins and direct photorepair, doublestranded gap repair and nucleotide excision repair systems (Crowley et al. 2006; Boubriak et al. 2008; Karan et al. 2014). 
Another haloarchaeal strain relevant to astrobiology, Halorubrum lacusprofundi, was isolated from Deep Lake in the Vestfold Hills of Antarctica (Franzmann et al. 1988). Deep Lake is perennially cold, with the temperature remaining subzero for more than 6 months of the year. However, Deep Lake does not freeze, even when temperatures drop to $-18^{\circ} \mathrm{C}$ due to freezing-point depression from high $(28 \%)$ salinity. H. lacusprofundi is capable of growth down to $-2^{\circ} \mathrm{C}$ and is well-adapted to this environment (Reid et al. 2006). $H$. lacusprofundi biofilms have been reported at the lowest growth temperatures and may serve to enhance survival. The $\mathrm{H}$. lacusprofundi genome has been completely sequenced and its proteins exhibit reduced surface acidity and internal amino acid substitutions characteristic of enhanced internal flexibility (Anderson et al. 2016; DasSarma et al. 2013; Karan et al. 2013).

In order to evaluate the astrobiological potential of Haloarchaea, we sought to compare the ability of Halobacterium sp. NRC-1 and H. lacusprofundi to survive trips into the stratosphere. While Earth's stratosphere exhibits multiple extremes not dissimilar to those found on the surface of Mars (Smith et al. 2011; Smith 2013), including cold temperatures, high radiation fluxes and low pressures, our primary interest in this study was to assess the importance of tolerance to extremely cold temperatures in survival. Our results show that while both halophiles are able to survive trips into the stratosphere, H. lacusprofundi exhibits greater viability and a more robust transcriptomic response in the extremely cold temperatures experienced.

\section{Materials and methods}

\section{Helium balloon launching and recovery}

Natural rubber $1.6 \mathrm{~kg}$ balloons filled with $5.66 \mathrm{~m}^{3}$ of helium were used to provide the free lift required to pull a $2.2 \mathrm{~kg}$ capsule to the stratosphere at a rate of approximately $300 \mathrm{~m}$ $\min ^{-1}$. Helium balloons were used for high-altitude launches from the Sierra Nevada Mountains of central California between February and July 2015. Items mounted on the outside of the payload included a cryogenic temperature probe, GPS 'SPOT' trackers and a GPS altimeter. Tubes containing Haloarchaea were affixed to the exterior of the payload in order to fully expose them to environmental conditions from ground level to altitudes of $33-36 \mathrm{~km}$ above sea level. When balloons reached peak altitude, they exploded and the payload plummeted towards Earth. A parachute slowed the descent, resulting in a soft landing. Each payload was recovered within about an hour of landing, by following the SPOT tracker GPS coordinates to the landing site.

\section{Haloarchaeal culturing and laboratory analysis}

Halobacterium sp. NRC-1 was cultured in $\mathrm{CM}^{+}$medium at $42^{\circ} \mathrm{C}$ while $H$. lacusprofundi ATCC 49239 was cultured in ATCC1682 Deep Lake medium at $30^{\circ} \mathrm{C}$, as previously described (Berquist et al. 2006; Reid et al. 2006; Anderson et al. 2016). Cultures were grown to late-log phase and aliquoted into cryovials for shipments by courier from
Maryland to California and launching into the stratosphere (Fig. 1). To determine colony forming units, cultures were diluted and plated on agar plates and colonies counted after 10 days incubation at the optimal growth temperatures. In each case, experiments were done in triplicate and standard deviations calculated using Microsoft Excel. Cells were photographed in a Nikon Labophot phase contrast microscope using a Nikon D90 DSLR. Pigment analysis was conducted after pelleting cells by centrifugation $(10 \mathrm{~min}, 10000 \times \boldsymbol{g})$, hypotonic lysis in deionized water and spectroscopy using a Shimadzu UV-1601 spectrophotometer. For viability assays after freeze-thaw, cultures in cryotubes were placed in a $-70^{\circ} \mathrm{C}$ freezer for $2 \mathrm{~h}$, followed by thawing at room temperature and determining colony forming units, as described above.

\section{Genome-wide transcriptomic analyses}

Custom whole genome DNA oligonucleotide microarrays for Halobacterium sp. NRC-1 and H. lacusprofundi were manufactured by Agilent Corporation (Coker et al. 2007). To compare transcriptional profiles, triplicate cultures of Halobacterium sp. NRC-1 grown at 42 or $15^{\circ} \mathrm{C}$ and $H$. lacusprofundi grown at 30 or $4^{\circ} \mathrm{C}$, both to an $\mathrm{OD}_{600}$ of 0.3 . The temperatures reflected either optimal or minimal growth temperatures for transcriptomic analysis. After collecting cells by centrifugation, RNA was isolated and pooled, $\mathrm{Cy} 3$ and Cy5-labelled cDNA was synthesized and labelled cDNA was hybridized to duplicate microarrays, washed and scanned on an Agilent DNA microarray scanner (model no. G2565BA), as previously described (Müller \& DasSarma 2005; Coker et al. 2007). Agilent Feature Extraction software was used for image analysis and processing of the microarray image file. Statistical analyses were performed as previously described using a 1.5 -fold change threshold cutoff. Expression data were aligned with protein families (conserved Haloarchaeal orthologous genes (cHOGs)) using MS Excel (DasSarma et al. 2010; Capes et al. 2012).

\section{Results}

\section{Flights into the stratosphere}

Halobacterium sp. NRC-1 and H. lacusprofundi grown in liquid cultures in the laboratory in Maryland were aliquoted and shipped to California by overnight courier (Fig. 1). Experimental samples were launched to the stratosphere affixed to the payloads of Earth to Sky Calculus high-altitude helium balloons while control aliquots were either stored on the ground in Maryland or shipped to and returned from California without balloon flights. Experimental vials were launched to heights of $36 \mathrm{~km}$, with flight times of $2.5 \mathrm{~h}$ from launch to landing, including $1 \mathrm{~h}$ in the stratosphere. The minimum temperature recorded was $-65^{\circ} \mathrm{C}$, with UV-C doses of $18 \mathrm{~J} \mathrm{~m}^{-2}$ and air pressure as low as $230 \mathrm{~Pa}$ (Jacob 1999). Samples were recovered typically within an hour and shipped by overnight courier to Maryland for analysis.

Experimental and control samples of Halobacterium sp. NRC-1 and $H$. lacusprofundi were analysed visually, 


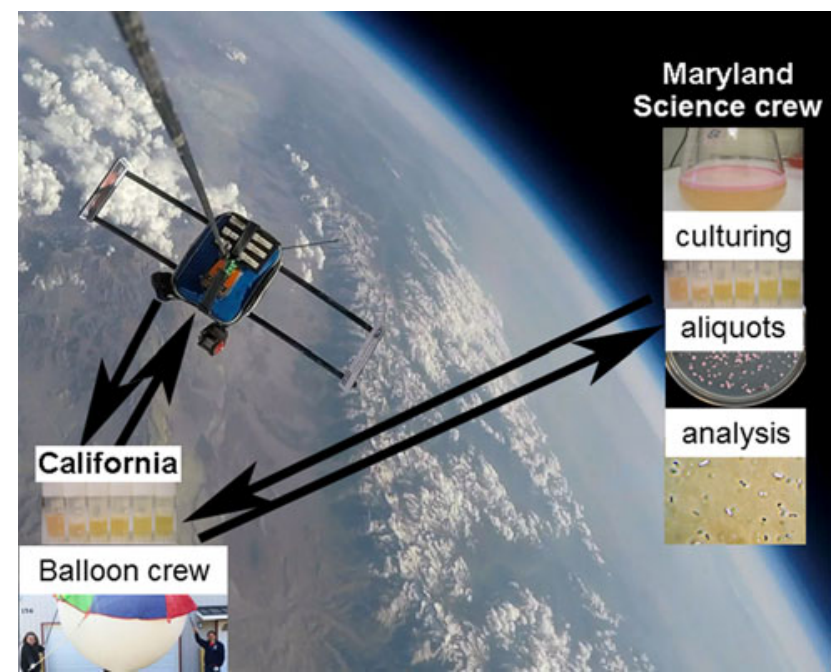

Fig. 1. Experimental coordination of balloon launches and Haloarchaeal microbiology. Cultures of Halobacterium sp. NRC-1 and $H$. lacusprofundi were grown in the University of Maryland and sent by courier to Earth to Sky Calculus for launching into the stratosphere. Samples were returned to Maryland for analysis.

spectrophotometrically and microscopically. Balloon-flown samples were found to be partially cleared and bleached upon visual inspection compared with the courier flown and ground-bound control samples. Examination of cells by phase contrast microscopy indicated that Halobacterium sp. NRC-1 cells had lost their refractive gas vesicles (data not shown), which normally provide buoyancy and promote cell flotation for phototrophic growth in their hypersaline environment (DasSarma et al. 2012). Morphological differences were less pronounced for $H$. lacusprofundi cells, which naturally lack gas vesicles and exhibit a higher level of pigmentation. The partial loss of pigmentation for both organisms after flights to the stratosphere was confirmed by UV-VIS spectroscopy, with reduced absorption detected at wavelengths characteristic of bacterioruberins (Fig. 2).

\section{Survival difference after stratospheric flights corresponds to freeze-thaw tolerance}

In order to determine the survival of Halobacterium sp. NRC-1 and $H$. lacusprofundi, dilutions of cultures remaining in Maryland and cultures that were shipped to and from California with or without experiencing the stratosphere from launches on balloons, were plated in triplicate. Cultures with or without shipping showed no statistical difference in survival, indicating that there was no significant effect on viability of transit by courier (Fig. 3). Interestingly, cultures of both Halobacterium sp. NRC-1 and H. lacusprofundi flown to the stratosphere appeared to show a significant degree of survival, although the Antarctic species showed relatively better survival than the mesophile (Fig. 3). Quantitative analysis indicated $50 \%$ survival for $H$. lacusprofundi and $5 \%$ for Halobacterium sp. NRC-1.

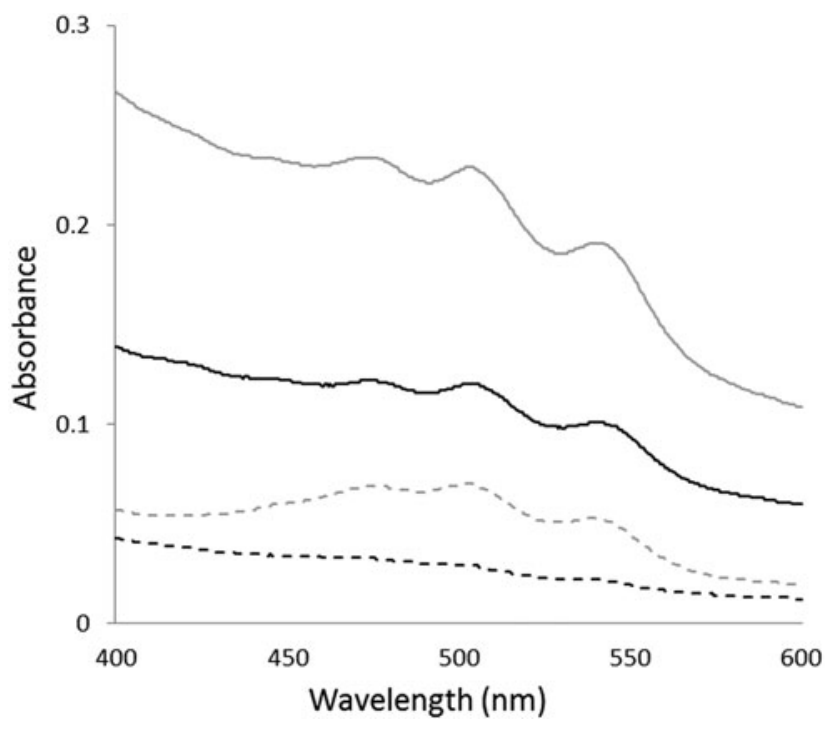

Fig. 2. Visible spectra of Halobacterium sp. NRC-1 (dashed) and $H$. lacusprofundi (solid) lysates, either balloon flown (black) or shipped by courier (gray).

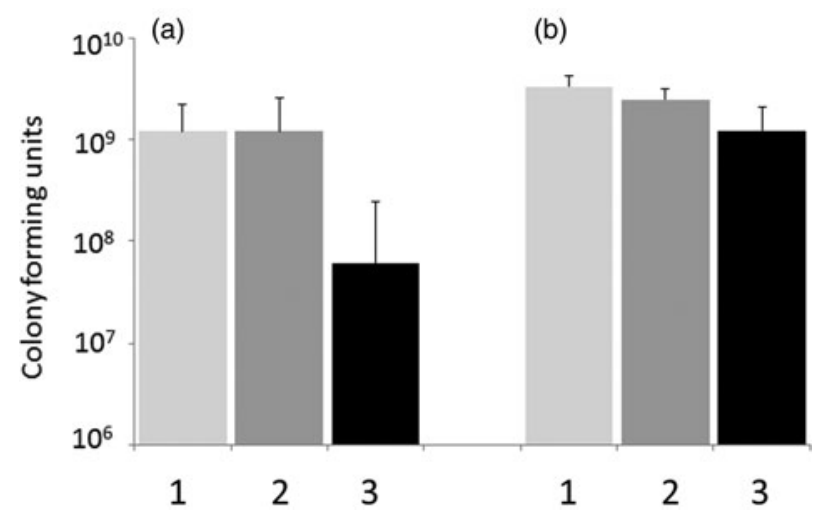

Fig. 3. Survival analysis of Halobacterium sp. NRC-1 (A) and $H$. lacusprofundi (B). Colony forming units are indicated on the $y$-axis for cultures remaining on the ground (1), courier transported (2) and exposed to the stratosphere (3). Error bars indicate standard deviations of three measurements.

In order to determine whether freeze-thaw tolerance was responsible for the observed difference in survival, we froze both Halobacterium sp. NRC-1 and H. lacusprofundi cultures in the laboratory. After thawing, survival of each Haloarchaeaon was quantified by serial dilution and plating. H. lacusprofundi was found to be considerably more tolerant to freeze-thaw than Halobacterium sp. NRC-1 (88 versus 16\% survival, respectively) (Fig. 4). These findings showed that the better freezethaw survival of the cold-adapted Antarctic isolate, H. lacusprofundi, compared with the mesophilic Halobacterium sp. NRC-1, parallels the observed higher survival in the stratosphere (Fig. 3). Interestingly, freeze-thaw also led to partial loss of pigmentation in both Haloarchaea and loss of intracellular gas vesicles in Halobacterium sp. NRC-1 (data not shown). 


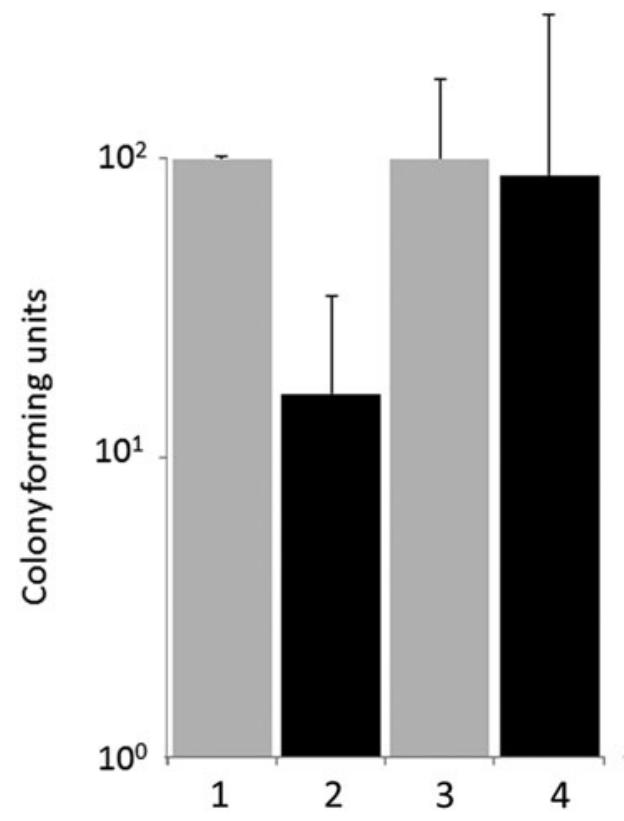

Fig. 4. Survival analysis of Halobacterium sp. NRC-1 and $H$. lacusprofundi exposed to freeze-thaw in the laboratory. Ambient temperature (gray) versus freeze-thawed (black) samples. Halobacterium sp. NRC-1 (1 and 2) and H. lacusprofundi (3 and 4). Error bars indicate standard deviations of three measurements.

\section{Genome-wide transcriptomic analysis}

In order to address the difference in cold-survival of Halobacterium sp. NRC-1 and H. lacusprofundi at the transcriptomic level, we compared genome-wide transcriptomes of the two microorganisms at low, versus optimum growth temperatures using DNA microarrays. For Halobacterium sp. NRC-1, DNA microarrays comparing the optimal temperature of $42^{\circ} \mathrm{C}$ to the low temperature of $15^{\circ} \mathrm{C}$ had been conducted earlier (Coker et al. 2007). We compared these data to transcriptomic data for $H$. lacusprofundi near its optimal growth temperature of $30^{\circ} \mathrm{C}$ and low growth temperature of $4^{\circ} \mathrm{C}$. The numbers of genes with significantly changed transcription levels are shown in Table 1.

The findings showed that gene expression in H. lacusprofundi is much more responsive to temperature differences than in Halobacterium sp. NRC-1 (Table 1). Nearly $60 \%$ of $H$. lacusprofundi genes were up- or down-regulated by $\geq 1.5$ fold in this cold-adapted haloarchaeon with nearly equal numbers of genes changing in each direction, $31 \%$ up-regulated and $29 \%$ down-regulated. By contrast, $<20 \%$ of genes in the mesophile Halobacterium sp. NRC-1 were regulated to the same extent, with twice as many down-regulated as up-regulated genes. Down-regulated genes are likely primarily growth rate-dependent since colder temperatures slow growth (Coker et al. 2007).

Regulated genes in the two halophiles were determined for conserved genes using the cHOGs database (DasSarma et al. 2010; Capes et al. 2011, 2012). The results for the subset of cHOGs were very similar to the genome as a whole, with $55 \%$ of $H$. lacusprofundi compared with $20 \%$ of
Halobacterium sp. NRC-1 orthologs significantly changed (Table 1). Most of the up-regulated cHOGs in Halobacterium sp. NRC-1 were also similarly perturbed in H. lacusprofundi (29 out of 43). Interestingly, some of the most highly up-regulated genes were conserved transcriptional regulators, general transcription factors and chaperones (Table 2). For transcription factors and regulators, there were a larger number of genes up-regulated in $H$. lacusprofundi compared with Halobacterium sp. NRC-1, while the number was nearly the same for chaperones. Orthologs of nearly all of the perturbed Halobacterium sp. NRC-1 genes were also found to be changed in $H$. lacusprofundi. These findings are consistent with a common core response to cold-temperature, as reflected by changes observed in both Haloarchaea, with an additional strong and diverse response to cold and freezing conditions specifically in the Antarctic H. lacusprofundi.

\section{Discussion}

Halophilic Archaea have been proposed to be excellent candidates for astrobiology (DasSarma 2006). We tested effects of stratospheric conditions on survival of live, metabolically active Haloarchaea by launching two species on helium balloons. Our results showed that both species, Halobacterium sp. NRC1 and $H$. lacusprofundi, can survive stratospheric flights, with the latter cold-adapted Antarctic species displaying superior survival ability compared with the former mesophilic species. $H$. lacusprofundi therefore appears better suited for stratospheric conditions.

Enhanced survival of $H$. lacusprofundi over Halobacterium sp. NRC-1 in the stratosphere is primarily due to its better adaptation to cold temperatures (Fig. 4). The stratosphere is very cold, between -10 and $-65^{\circ} \mathrm{C}$ (Jacob 1999), with the lower temperature resulting in freezing of the hypersaline culture media. Upon return to the troposphere or after landing, Haloarchaeal cultures thawed. Since H. lacusprofundi experiences frigid subzero temperatures for 8 months of the year in its natural environment of Deep Lake in the Vestfold Hills of Antarctica, it is better capable of tolerating the cold freeze occurring in the stratosphere (Franzmann et al. 1988; Reid et al. 2006). In contrast, the mesophilic species isolated from a temperate climate does not appear to be able to withstand cold temperatures well and loses considerably more viability from the journey into the stratosphere (Coker et al. 2007).

The cold-adaptive mechanisms operating in $H$. lacusprofundi and Halobacterium sp. NRC-1 were found to have similarities and differences using genome-wide transcriptomic analysis (Tables 1 and 2) (Coker et al. 2007). H. lacusprofundi exhibited significantly higher percentage and numbers of cold-induced genes, including general transcription factors and transcriptional regulators. In Halobacterium sp. NRC-1, our prior work had shown that three transcription factors (TbpD, $\mathrm{TfbA}$ and $\mathrm{TfbG}$ ) were involved in, or were responsive to cold temperatures, with only a single one, $\mathrm{TfbG}$, displaying expression level perturbation by cold temperature (Coker \& DasSarma 2007; Coker et al. 2007). H. lacusprofundi showed five transcription factors with gene expression responsive to 
Table 1. Genes perturbed by low temperatures in Halobacterium $s p$. NRC-1 and $\mathrm{H}$. lacusprofundi

\begin{tabular}{llccr}
\hline & Total no. of genes & Up-regulated (\%) & Down-regulated (\%) & Total up- or down-regulated (\%) \\
\hline Halobacterium sp. NRC-1 $\left(42\right.$ versus $\left.15^{\circ} \mathrm{C}\right)$ & 2484 & $168(6.76)$ & $310(12.48)$ & $478(19.24)$ \\
H. lacusprofundi $\left(30\right.$ versus $\left.4{ }^{\circ} \mathrm{C}\right)$ & 3562 & $1113(31.16)$ & $1024(28.75)$ & $2137(59.99)$ \\
Halobacterium sp. NRC-1 genes in cHOGs & 841 & $43(5.11)$ & $127(15.10)$ & $170(20.21)$ \\
H. lacusprofundi genes in cHOGs & 871 & $247(28.36)$ & $233(26.75)$ & $480(55.11)$ \\
Number of cHOGs in both haloarchaea & 784 & $29(3.70)$ & $27(3.44)$ & $56(7.14)$ \\
\hline
\end{tabular}

Table 2. Selected cold up-regulated genes in Halobacterium sp. $N R C-1$ and $\mathrm{H}$. lacusprofundi

\begin{tabular}{|c|c|c|c|c|c|c|c|}
\hline \multirow{2}{*}{ Gene type and annotation } & \multirow[b]{2}{*}{$\mathrm{HOG}$} & \multicolumn{3}{|c|}{ Halobacterium sp. NRC-1 } & \multicolumn{3}{|c|}{ H. lacusprofundi } \\
\hline & & Gene ID & Change & $P$-value & Gene ID & Change & $P$-value \\
\hline \multicolumn{8}{|l|}{ Transcription factors } \\
\hline TBP TATA-box binding family protein & & & & & Hlac_2818 & 2.91 & 0.01 \\
\hline TBP TATA-box binding family protein & 44 & & & & Hlac_3413 & 2.09 & 0.48 \\
\hline TFB transcription initiation factor IIB & 18 & $t f b \mathrm{G}$ & 2.26 & 0.26 & Hlac_1495 & 3.60 & 0.00 \\
\hline TFB transcription initiation factor IIB & 4 & & & & Hlac_1513 & 6.27 & 0.00 \\
\hline TFB transcription initiation factor IIB & 4 & & & & Hlac_601 & 2.05 & 0.00 \\
\hline \multicolumn{8}{|l|}{ Transcriptional regulators } \\
\hline Metal dependent repressor, DtxR family & 624 & $\operatorname{sir} \mathrm{R}$ & 3.75 & 0.21 & Hlac_657 & 2.08 & 0.31 \\
\hline Transcriptional regulator, ArsR family & 325 & boa2 & 1.55 & 0.12 & Hlac_1852 & 2.50 & 0.04 \\
\hline Transcriptional regulator, PadR family & 1 & vng751 & 1.80 & 0.00 & Hlac_3648 & 11.60 & 0.13 \\
\hline Transcriptional regulator, PadR family & 1 & & & & Hlac_3619 & 2.22 & 0.71 \\
\hline Transcriptional regulator, ArsR family & 94 & & & & Hlac_395 & 2.93 & 0.00 \\
\hline Transcriptional regulator, ArsR family & 731 & & & & Hlac_184 & 177.04 & 0.00 \\
\hline Transcriptional regulator, AsnC family & 731 & & & & Hlac_3617 & 2.12 & 0.78 \\
\hline Transcriptional regulator, AsnC family & 425 & & & & Hlac_2326 & 2.47 & 0.43 \\
\hline Transcriptional regulator, CopG family & 109 & & & & Hlac_2067 & 3.57 & 0.00 \\
\hline Transcriptional regulator, CopG family & 977 & & & & Hlac_524 & 3.35 & 0.00 \\
\hline Transcriptional regulator, TrmB family & 43 & & & & Hlac_2144 & 2.10 & 0.13 \\
\hline Transcriptional regulator, XRE family & 632 & & & & Hlac_273 & 3.99 & 0.05 \\
\hline \multicolumn{8}{|l|}{ Stress proteins and chaperones } \\
\hline Cold-shock protein & 2 & $\operatorname{csp} \mathrm{D} 2$ & 3.11 & 0.02 & Hlac_1630 & 4.80 & 0.00 \\
\hline Cold-shock protein & 2 & $\operatorname{cspD} 1$ & 2.31 & 0.44 & Hlac_1312 & 2.16 & 0.00 \\
\hline Small heat shock protein (HSP20 family) & 237 & vng283 & 1.81 & 0.07 & Hlac_637 & 1.95 & 0.00 \\
\hline Thermosome, GroEL (HSP60 family) & 320 & $\operatorname{cct} \mathrm{B}$ & 1.79 & 0.01 & Hlac_416 & 2.74 & 0.00 \\
\hline Prefoldin, alpha subunit & 428 & vng2465 & 1.68 & 0.00 & Hlac_822 & 3.10 & 0.00 \\
\hline Prefoldin, beta subunit & 403 & & & & Hlac_567 & 15.21 & 0.00 \\
\hline 20S proteasome A and B subunits & 127 & & & & Hlac_608 & 2.06 & 0.01 \\
\hline Predicted ATP-dependent protease & 200 & lon & 1.48 & 0.25 & & & \\
\hline
\end{tabular}

cold temperatures, two Tbps and three Tfbs. Similarly, H. lacusprofundi also exhibited a larger number of transcriptional regulators responding to cold temperatures, twelve versus only three in Halobacterium sp. NRC-1. For stress protein and chaperone genes, five orthologous genes were up-regulated in both, with only three non-orthologs varying between the two (two in H. lacusprofundi and one in Halobacterium sp. NRC1). Together, these findings showed that while the core coldstress response is quite similar in both Haloarchaea, the Antarctic halophile has a much larger and diverse set of cold-responsive genes compared with the mesophile. These findings likely account for $H$. lacusprofundi's better survival under cold and freezing temperatures encountered in the stratosphere.

This is the first study of survival of live Haloarchaeal cultures under stratospheric conditions. Prior studies have examined the survival ability of a related species, Halorubrum chaoviatoris, from a Mexican intertidal marine area during a flight aboard ESA's Biopan 1 facility (Mancinelli et al. 1998). This experiment exposed desiccated cells to deep space conditions with considerably higher dose of radiation, leading to lowering of viability by $10^{6}$-fold. Clearly, exposure of desiccated cells to space conditions is significantly more challenging for the microbes. The current study is more likely to reflect potential survival of Haloarchaea under low temperatures, in surface or subsurface brines, than unprotected exposure to the surface of Mars or deep space. Additional studies are needed to determine the combinatorial effects of several stressors, including cold temperature, UV-C and cosmic radiation and low pressures.

A variety of other microorganisms have been tested for survival under space and simulated Mars conditions and in the stratosphere. In one study of nearly two-dozen bacterial species exposed to cold, low pressure and anoxic conditions in the 
laboratory, only a single microbe, Serratia liquefaciens was found to survive all of the extremes (Schuerger et al. 2013). In this study, two extremophiles, Deinococcus radiodurans and Psychrobacter cryohalolentis, were not capable of survival under these conditions. Other studies have focused on spore forming bacteria, such as Bacillus subtilis (Nicholson et al. 2012; Wassmann et al. 2012) and B. pumilus SAFR-032. The quiescent spore forms of $B$. pumilus are highly resistant to UV radiation and cold temperatures (Calcott \& MacLeod 1975; Vaishampayan et al. 2012).

Recently, several commercial bacterial cultures (Staphylococcus aureus, methicillin-resistant Staphylococcus aureus, Escherichia coli and Salmonella typhimurium) and humanassociated bacteria (Proteus mirabilis and Pseudomonas aeruginosa) were launched into the stratosphere on helium balloons (Chudobova et al. 2015). The survival of these bacteria was found to be significantly poorer than for the Haloarchaea in our study, especially H. lacusprofundi. Moreover, this study reported survival to be lower in the stratosphere than for a similar dose exposure to UV-C in the laboratory. However, the effects of temperature were not investigated in this study.

While the focus in our study has been survival in cold temperatures, we have also examined the effect of UV-C on the two Haloarchaea in the past (McCready et al. 2005; Crowley et al. 2006; Boubriak et al. 2008; our unpublished data). Our findings have shown that Halobacterium sp. NRC-1 and $\mathrm{H}$. lacusprofundi are both quite tolerant to UV-C, with an $\mathrm{LD}_{50}$ of $\sim 50 \mathrm{~J} \mathrm{~m}^{-2}$. Additional experiments are needed to determine the relative importance of UV-C, low pressure and other stressors in the partial loss of viability of Haloarchaea in the stratosphere. However, such studies would require longer flights.

\section{Conclusions}

Halobacterium sp. NRC-1 and H. lacusprofundi are able to withstand exposure to stratospheric conditions. H. lacusprofundi, an isolate from a perennially cold, hypersaline lake in Antarctica retains greater viability, at least for the time periods experienced using helium balloons, a property likely resulting from better cold and freeze adaptation. Most of the genes in the genome of the Antarctic species are significantly regulated at cold temperatures, which likely results in its better adaptation to the cold conditions in its natural environment and in the stratosphere.

\section{Acknowledgements}

Our work was supported by National Aeronautics and Space Administration grant NNX15AM07 G to S.D. We thank Sam Johnson, Justin Gilpin, Michael White, Bronwyn Stephenson and Jordan Herbst for assistance with balloon flights.

\section{Author Disclosure Statement}

No competing financial interests exist.

\section{References}

Anderson, I.J., DasSarma, P., Lucas, S., Copeland, A., Lapidus, A., Del Rio, T.G., Tice, H., Dalin, E., Bruce, D.C., Goodwin, L. et al. (2016). Complete genome sequence of the Antarctic Halorubrum lacusprofundi type strain ACAM 34. Stand. Genomic Sci. 11, 70.

Berquist, B.R., Müller, J.A. \& DasSarma, S. (2006). Genetic Systems for Halophilic Archaea. In Methods in Microbiology, ed. Oren, A. \& Rainey, F., pp. 637-668. Elsevier/Academic Press.

Boubriak, I., Ng, W.L., DasSarma, P., DasSarma, S., Crowley, D.J. \& McCready, S.J. (2008). Transcriptional responses to biologically relevant doses of UV-B radiation in the model archaeon, Halobacterium sp. NRC1. Saline Syst. 4, 13.

Calcott, P.H. \& MacLeod, R.A. (1975). The survival of Escherichia coli from freeze-thaw damage: permeability barrier damage and viability. Can. J. Microbiol. 21, 1724-1732.

Capes, M.D., Coker, J.A., Gessler, R., Grinblat-Huse, V., DasSarma, S.L., Jacob, C.G., Kim, J.M., DasSarma, P. \& DasSarma, S. (2011). The information transfer system of halophilic archaea. Plasmid 65, 77-101.

Capes, M.D., DasSarma, P. \& DasSarma, S. (2012). The core and unique proteins of haloarchaea. BMC Genomics 13, 39.

Chudobova, D., Cihalova, K., Jelinkova, P., Zitka, J., Nejdl, L., Guran, R., Klimanek, M., Adam, V. \& Kizek, R. (2015). Effects of stratospheric conditions on the viability, metabolism and proteome of prokaryotic cells. Atmosphere 6, 1290-1306.

Coker, J.A. \& DasSarma, S. (2007). Genetic and transcriptomic analysis of transcription factor genes in the model halophilic Archaeon: coordinate action of TbpD and TfbA. BMC Genet.. 8, 61 .

Coker, J.A., DasSarma, P., Kumar, J., Müller, J.A. \& DasSarma, S. (2007). Transcriptional profiling of the model Archaeon Halobacterium sp. NRC1: responses to changes in salinity and temperature. Saline Syst. 3, 6.

Crowley, D.J., Boubriak, I., Berquist, B.R., Clark, M., Richard, E., Sullivan, L., DasSarma, S. \& McCready, S. (2006). The $u v r A$, uvrB, and $u v r C$ genes are required for repair of ultraviolet light induced DNA photoproducts in Halobacterium sp. NRC-1. Saline Syst. 2, 11.

DasSarma, S. (2006). Extreme halophiles are models for astrobiology. Microbe 1, 120-127.

DasSarma, S. \& DasSarma, P. (2012). Halophiles. In eLS, John Wiley \& Sons, Ltd, DOI: 10.1002/9780470015902.a0000394.pub3.

DasSarma, S. \& DasSarma, P. (2015). Halophiles and their enzymes: negativity put to good use. Curr. Op. Microbiol. 25, 120-126.

DasSarma, S.L., Capes, M.D., DasSarma, P. \& DasSarma, S. (2010). HaloWeb: the haloarchaeal genomes database. Saline Syst. 6, 12.

DasSarma, S., Berquist, B.R., Coker, J.A., DasSarma, P. \& Müller, J.A. (2006). Post-genomics of the model haloarchaeon Halobacterium sp. NRC-1. Saline Syst. 2, 3

DasSarma, P., Zamora, R.C., Müller, J.A. \& DasSarma, S. (2012). Genomewide responses of the model archaeon Halobacterium sp. strain NRC-1 to oxygen limitation. J. Bacteriol. 194, 5520-5537.

DasSarma, S., Capes, M.D., Karan, R. \& DasSarma, P. (2013). Amino acid substitutions in cold-adapted proteins from Halorubrum lacusprofundi, an extremely halophilic microbe from Antarctica. PLoS ONE 8, e58587.

DeVeaux, L.C., Müller, J.A., Smith, J., Petrisko, J., Wells, D.P. \& DasSarma, S. (2007). Extremely radiation-resistant mutants of a halophilic archaeon with increased single-stranded DNA binding protein (RPA) gene expression. Radiation Res. 168, 507-514.

Franzmann, P.D., Stackebrandt, E., Sanderson, K., Volkman, J.K., Cameron, D.E., Stevenson, P.L., McMeekin, T.A. \& Burton, H.R. (1988). Halobacterium lacusprofundi sp. nov., a halophilic bacterium isolated from Deep Lake, Antarctica. Syst. Appl. Microbiol. 11, 20-27.

Goh, F., Jeon, Y.J., Barrow, K., Neilan, B.A. \& Burns, B.P. (2011). Osmoadaptive strategies of the archaeon Halococcus hamelinensis isolated from a hypersaline stromatolite environment. Astrobiology 11, 529-536.

Horneck, G., Klaus, D.M. \& Mancinelli, R.L. (2010). Space microbiology. Microbiol Mol. Biol. Rev. 74, 121-156.

Jacob, D.J. (1999). Introduction to Atmospheric Chemistry. Princeton University Press, USA. 
Karan, R., Capes, M.D. \& DasSarma, S. (2012). Function and biotechnology of extremophilic enzymes in low water activity. Aquatic Biosyst. 8, 4.

Karan, R., Capes, M.D. \& DasSarma, S. (2013). Cloning, overexpression, purification, and characterization of a polyextremophilic $\beta$-galactosidase from the Antarctic haloarchaeon Halorubrum lacusprofundi. BMC Biotechnol. 13, 3.

Karan, R., DasSarma, P., Balcer-Kubiczek, E., Weng, R.R., Liao, C.C., Goodlett, D.R., Ng, W.V. \& DasSarma, S. (2014). Bioengineering radioresistance by overproduction of RPA, a mammalian-type singlestranded DNA-binding protein, in a halophilic archaeon. Appl. Microbiol. Biotechnol. 98, 1737-1747.

Kounaves, S.P., Chaniotakis, N.A., Chevrier, V.F., Carrier, B.L., Folds, K. E., Hansen, V.M., McElhoney, K.M., O’Neil, G.D. \& Weber, A.W. (2014). Identification of the perchlorate parent salts at the Phoenix Mars landing site and possible implications. Icarus 232, 226-231.

Leuko, S., Rettberg, P., Pontifex, A.L. \& Burns, B.P. (2014). On the response of halophilic archaea to space conditions. Life (Basel) 4, 66-76.

Mancinelli, R.L., White, M.R. \& Rothschild, L.J. (1998). Biopan-survival I: exposure of the osmophiles Synechococcus sp. (Nageli) and Haloarcula sp. to the space environment. Adv. Space Res. 22, 327-334.

Mancinelli, R.L., Fahlen, T.F., Landheim, R. \& Klovstad, M.R. (2004). Brines and evaporites: analogs for Martian life. Adv. Space Res. 33, 1244-1246.

McCready, S., Müller, J.A., Boubriak, I., Berquist, B.R., Ng, W.L. \& DasSarma, S. (2005). UV irradiation induces homologous recombination genes in the model archaeon, Halobacterium sp. NRC-1. Saline Syst. 1, 3.

McEwen, A.S., Ojha, L., Dundas, C.M., Mattson, S.S., Byrne, S., Wray, J.J., Cull, S.C., Murchie, S.L., Thomas, N. \& Gulick, V.C. (2011). Seasonal flows on warm Martian slopes. Science 333, 740-743.

Müller, J.A. \& DasSarma, S. (2005). Functional genomic analysis of anaerobic respiration of the archaeon Halobacterium sp. NRC-1: dimethyl sulfoxide (DMSO) and trimethylamine N-oxide (TMAO) as terminal electron acceptors. J. Bacteriol. 187, 1659-1667.

Nicholson, W.L., Moeller, R. \& Horneck, G. (2012). Transcriptomic responses of germinating Bacillus subtilis spores exposed to 1.5 years of space and simulated martian conditions on the EXPOSE-E experiment PROTECT. Astrobiology 12, 469-486.

Ojha, L., Wilhelm, M.B., Murchie, S.L., McEwen, A.S. \& Wray, J.J. (2015). Spectral evidence for hydrated salts in recurring slope lineae on Mars. Nat. Geosci. 8, 829-832.

Reid, I.N., Sparks, W.B., Lubow, S., McGrath, M., Livio, M., Valenti, J., Sowers, K.R., Shukla, H.D., MacAuley, S., Miller, T. et al. (2006). Terrestrial models for extraterrestrial life: methanogens and halophiles at Martian temperatures. Int. J. Astrobiol. 5, 89-97.

Rummel, J.D., Beaty, D.W., Jones, M.A., Bakermans, C., Barlow, N.G., Boston, P.J., Chevrier, V.F., Clark, B.C., de Vera, J.-P.P., Gough, R.V. et al. (2014). A New analysis of mars "Special Regions": findings of the second MEPAG special regions science analysis group (SR-SAG2). Astrobiology 14, 887-968.

Schuerger, A.C., Ulrich, R., Berry, B.J. \& Nicholson, W.L. (2013). Growth of Serratia liquefaciens under $7 \mathrm{mbar}, 0{ }^{\circ} \mathrm{C}$, and $\mathrm{CO}_{2}$-enriched anoxic atmospheres. Astrobiology 13, 115-131.

Smith, D.J. (2013). Microbes in the upper atmosphere and unique opportunities for astrobiology research. Astrobiology 13, 981-990.

Smith, D.L., Griffin, D.W., McPeters, R.D., Ward, P.D. \& Schuerger, A.C. (2011). Microbial survival in the stratosphere and implications for global dispersal. Aerobiologia 27, 319-332.

Stoker, C.R., Zent, A., Catling, D.C., Douglas, S., Marshall, J.R., Archer, D. Jr., Clark, B., Kounaves, S.P., Lemmon, M.T., Quinn, R. et al. (2010). Habitability of the Phoenix landing site. J. Geophys. Res. 115, E00E20.

Toner, J.D., Catling, D.C. \& Light, B. (2014). Soluble salts at the Phoenix lander site, Mars: a reanalysis of the wet chemistry laboratory data. Geochim. Cosmochim. Acta 136, 142-168.

Vaishampayan, P.A., Rabbow, E., Horneck, G. \& Venkateswaran, K.J. (2012). Survival of Bacillus pumilus spores for a prolonged period of time in real space conditions. Astrobiology 12, 487-497.

Wassmann, M., Moeller, R., Rabbow, E., Panitz, C., Horneck, G., Reitz, G., Douki, T., Cadet, J., Stan-Lotter, H., Cockell, C.S et al. (2012). Survival of spores of the UV-resistant Bacillus subtilis strain MW01 after exposure to low-earth orbit and simulated martian conditions: data from the space experiment ADAPT on EXPOSE-E. Astrobiology 12, 498-507. 